\title{
Les Nappes Profondes : \\ Ressources en eau d'ultime recours en cas de crise de pollution majeure
}

\author{
par Michel Mesny
}

du Ministère de l'Environnement, Mission Défense, Paris

\author{
par Jean-Pierre Comte
}

du BRGM, Centre thématique Eau, Montpellier

\section{INTRODUCTION}

En cas de crise de pollution majeure d'origine chimique, radioactive ou biologique, les eaux de surface et celles des nappes phréatiques superficielles peuvent être contaminées instantanément et rendues impropres à la consommation humaine. Or plus des trois quarts des eaux destinées à l'alimentation en eau potable proviennent de ces ressources vulnérables. Les nappes profondes apparaissent alors comme une ressource alternative, protégée de manière au moins temporaire, sinon permanente.
Cette eau de substitution doit satisfaire trois conditions : - elle doit pouvoir être mobilisée immédiatement et distribuée par les moyens appropriés (réseaux d'adduction existants, bornes-fontaines de quartier, embouteillage ou «ensachage »).

- les normes de potabilité doivent être quasi-respectées pendant une durée limitée, puis rapidement, totalement respectées.

- les volumes prélevés doivent répondre aux besoins minima de la population, puis augmenter au fur et à mesure du retour aux conditions normales, ou de la mise en place d'équipements supplémentaires (en production et en distribution).

Within the framework of a reflection dealing with the possibility to insure sufficient drinking water supply in case of masr crisis, the Ministry of the Environment undertook an inventon of the aquiferous spstems on the whole French (continental) territor): In case of a generalized surface water and groudwater contamina tion, these aquifers could provide substitution water, qualified as a last resorted water $)$ because of its temporary - fast definitive - protection statute.

A scale of value relative to the protection level was created, wich enables the researchers to identify three levels of protection and to draw up a $1 / 1,500,000$ scale mape of France, on wich the limits of 98 phreatic water-tables, identified as protected, have been reported. The great majorits of the aquifers corresponds to confined waters.

A stanistical analysis on the existence of protected resources reveats that 16 departments out of 96 have got protected resources on the whole territory, and that 22 others havent got any that are registrered at national scale. Otherwise, 67 towns out of 103 which count more than 50,000 inhabitants have got protected resources, which correspond to $61 \%$ of the urban population.

In a second time, the cases of the urban centres of Paris and Lvon will be looked into more in detail, stating precisely the protected resources which could be moblized and the existing collecting equipments which could possibly be integrated in a device used as a last resort. 


\section{II 圈 LES RESSOURCES PROTÉGÉES}

\subsection{Critères de définition de la protection}

La notion de protection résulte d'un décalage temporaire entre la date de pollution en surface et l'arrivée de la contamination au niveau du point de captage de l'eau. Elle dépend donc de la nature des terrains sus-jacents à l'aquifere (couverture plus ou moins imperméable), des caractéristiques hydrodyna- miques de l'aquifère (épaisseur, perméabilité), et des possibilités d'infiltrations polluantes (apports latéraux, failles, circulation karstique, ouvrages de pompage mal conçus ou réalisés). Le délai a été évalué entre un et trois mois.

Cette approche fondée sur l'évaluation du temps de transfert de la pollution dans le sous-sol ne présume pas de la durée effective de la pollution de l'ensemble du système de ressource « eau de surface» plus « eau souterraine ». Celleci met en jeu des phénomènes tels que ruissellement, infil-

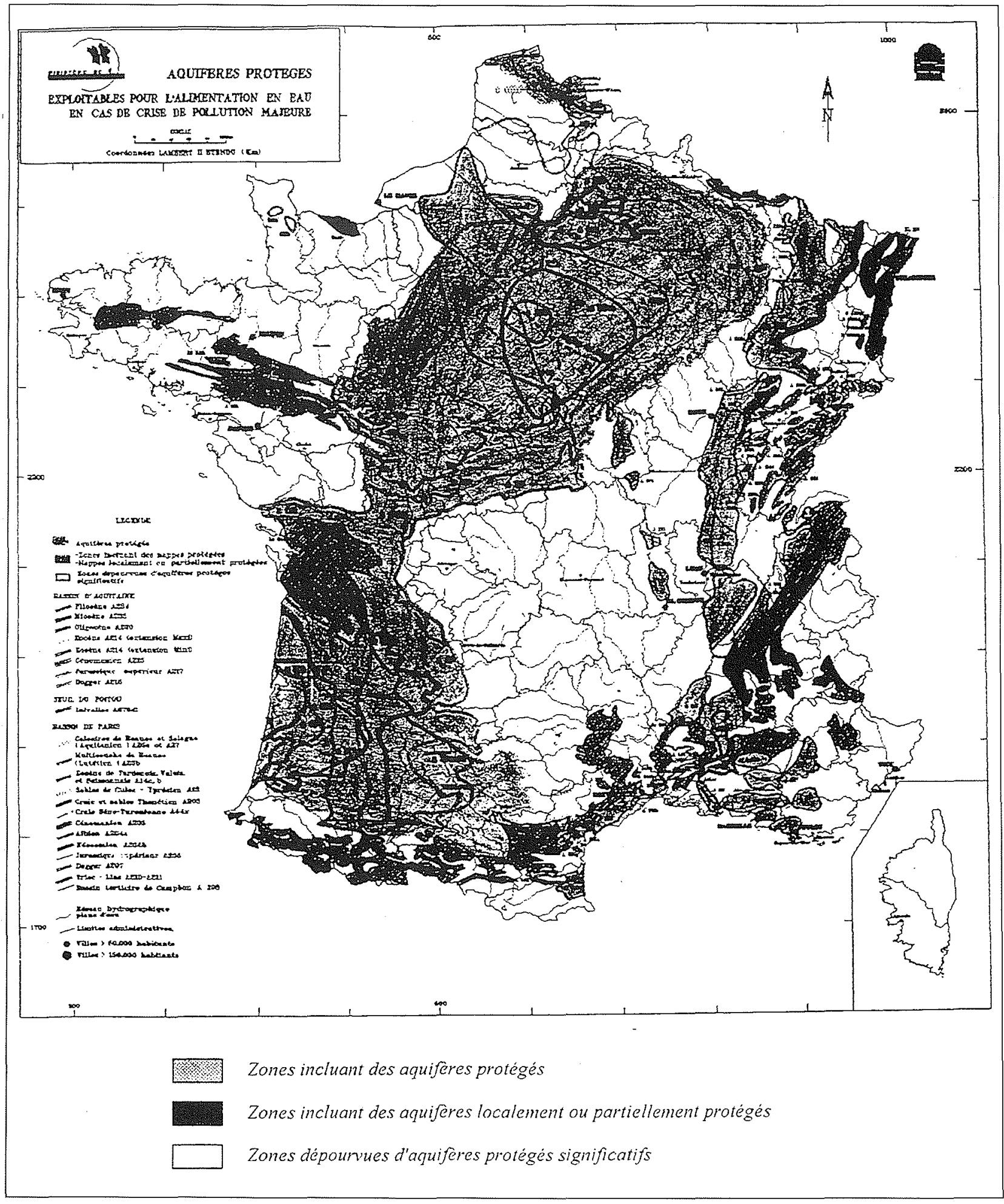

1. Carte de localisation des aquifères protégés. 
tration, diffusion, relargage, écoulements,... Ainsi, pour des études plus précises au niveau régional ou local, il conviendrait de considérer aussi :

- la nature et les caractéristiques du polluant (coefficient de diffusion, adsorption,...) ;

- la nature de l'impact et la vitesse de propagation de la pollution;

- l'évolution dans le temps et l'espace de la concentration de la pollution en surface.

Cette notion de protection de la ressource est par conséquent relative et évolutive. Elle est complétée par un critère d'exploitabilité prenant en compte l'accessibilité, la productivité et la qualité de l'aquifère, lesquelles sont fonction de la géométrie, des caractéristiques hydrodynamiques et des faciès hydrochimiques rencontrés.

Trois classes relatives au degré de protection des territoires ont été définies pour l'inventaire national

- classe A correspondant aux territoires disposant d'au moins un système de ressource protégée exploitable ;

- classe B correspondant aux territoires ne disposant que partiellement de systèmes de ressources protégées exploitables (nappes reconnues en partie protégées, nappes protégées mais non reconnues, nappes protégées mais non identifiables à l'échelle nationale);

- classe $\mathbf{C}$ correspondant aux territoires ne disposant pas de système de ressource protégée exploitable.

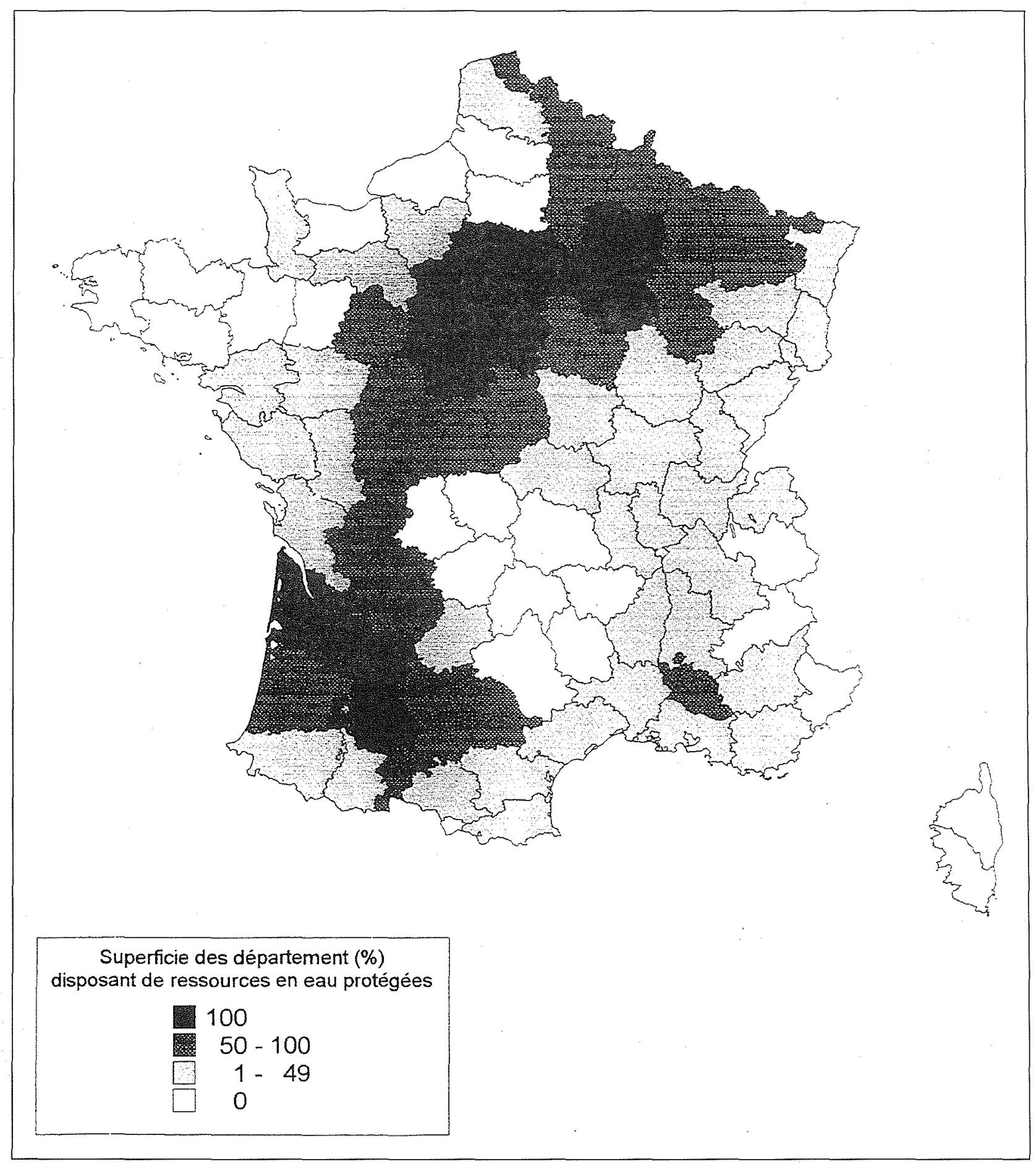

2. Carte de répartition des ressources protégées par département. 


\section{解 2.2 Localisation des aquifères protégés (fig. 1)}

Les différents schémas de protection des aquifères, croisés aux différents processus de contamination, définissent des situations régionales très contrastées.

Un très grand domaine continu de classe A ressort de l'inventaire national: il est constitué par le Bassin Parisien et le Bassin Aquitain reliés par le seuil du Poitou. Caractérisés par des structures synclinales, ces bassins foumissent des ressources en eau protégée à partir des formations stratigraphiques les plus anciennes à leur périphérie (Trias, Jurassique moyen et supérieur) et de plus en plus récentes au fur et à mesure de leur ennoiement sous l'épaisseur croissante des couches vers le centre (Crétacé dont l'Albien du Bassin Parisien, et Tertiaire). La profondeur et la salinité des eaux sont des facteurs limitants de l'exploitation des aquifères contenus dans les formations les plus anciennes au centre de ces bassins. Entre ces deux bassins, le seuil du Poitou dispose d'un aquifère protégé dans les calcaires infraliasiques.

En dehors de ce domaine, le Nord-Pas-de-Calais dispose d'importantes ressources protégées en eaux souterraines de classe A (calcaires fissurés du Carbonifère et craie d'Artois), ainsi que la Lorraine (grès du Trias) et la Bourgogne (Trias, Dogger, Malm et Miocène).

Les régions de Franche-Comté, Rhône-Alpes, ProvenceAlpes-Côte d'Azur et Languedoc-Roussillon possèdent aussi des ressources protégées de classe A mais leur étendue est souvent limitée et les aquifères sont très compartimentés. fiés.

Au total. 98 systèmes protégés de classe A ont été identi-

Les zones de ressources protégées de classe B correspondent essentiellement au Massif Armoricain, aux Pyrénées, aux Alpes, au Jura et aux Causses cévenols où les aquifères sont très localisés et discontinus, ou bien mal connus. Par ailleurs, les aquifères du Dogger et du Malm de Poitou-Charentes sont a priori protégés mais ils sont très peu connus. La nappe phréatique de la plaine d'Alsace a aussi été considérée comme faisant partie de la classe B à cause de son épaisseur. Ces systèmes protégés de classe B sont au nombre de 26.

Il n'y a pas de ressources protégées en eaux souterraines (classe C) dans les zones de socle du Massif Armoricain, du Massif Central, des Pyrénées, des Alpes, de la Corse et des Vosges, ou dans les zones à couverture sédimentaire à nappe libre uniquement (Normandie, Artois, Picardie, une partie de la Lorraine et de la Bourgogne,...).

\section{RÉPARTITION DES RESSOURCES PROTÉGÉES}

La répartition des ressources protégées en eaux souterraines vis-à-vis de la population a été établie selon deux approches:

- l'une globale, en calculant le pourcentage de la surface des départements de France métropolitaine couvert par des territoires pourvus d'aquifères protégés ;

- l'autre ciblée sur les populations urbaines des villes de plus de 50000 habitants.

\subsection{Répartition par département (fig. 2)}

Sur les 96 départements métropolitains :

- 16 disposent d'aquifères protégés sur la totalité de leur territoire : il s'agit des départements centraux du Bassin Parisien et du Bassin Aquitain.

- 20 disposent d'aquifères protégés sur plus de la moitié de leur territoire : il s'agit des départements situés à la périphérie du Bassin Parisien et du Bassin Aquitain, auxquels s'ajoutent le Nord et le Vaucluse.

- 38 disposent d'aquifères protégés sur moins de la moitié de leur territoire: il s'agit des départements d'Alsace.
Franche-Comté, Bourgogne, Languedoc-Roussillon, et en partie Rhône-Alpes, Provence-Alpes-Côte d'Azur, Pyrénées, Poitou-Charentes, Pays de la Loire et Basse-Normandie.

- 22 ne disposent pas de ressources protégées, du moins identifiées à l'échelle nationale: il s'agit du Massif Armoricain, du Massif Central et d'une partie des Alpes, de la Haute-Normandie et de la Picardie.

\subsection{Répartition par ville de plus de $\mathbf{5 0} 000$ habitants} (fig. 3)

Les 103 villes de plus de 50000 habitants rassemblent environ 13,2 millions de personnes dont:

- 8 millions disposent de ressources protégées (soit $61 \%$ ) dans 61 villes;

- 0.8 millions disposent de ressources potentiellement protégées (soit $6 \%$ ) dans 6 villes ;

- 4,4 millions ne disposent pas de ressources protégées (soit $33 \%$ ) dans 36 villes.

Répartition (\%) des ressources protégées pour les villes de plus de 50000 habitants

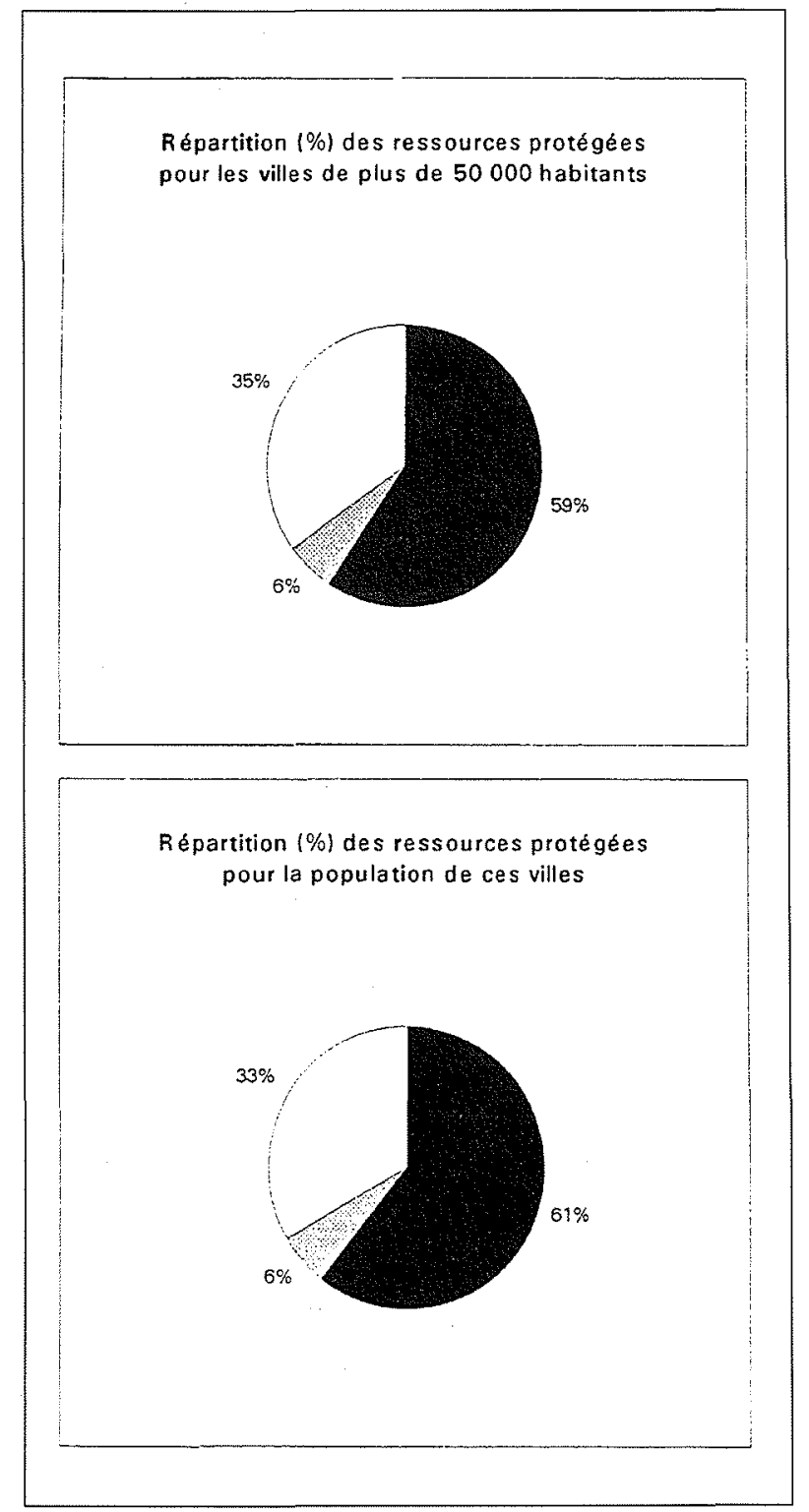

3. Répartition des ressources protégées vis-à-vis des populations urbaines. 